Artículo

\title{
Innovaciones tecnológicas en Bibliotecas
}

\section{Technological innovations in Libraries}

\author{
Ana Ruth Díaz Vásquez \\ Regina Romero de De la Vega \\ Dina Marjorie Rodas Escobar \\ Aura Marina Ramírez Cortez \\ Mariana de Jesús Cabrera Morales \\ Maestría en Bibliotecología y Ciencia de la Información \\ Universidad de San Carlos de Guatemala \\ Correo para recibir correspondencia: aruthdiaz@gmail.com
}

\section{$\varangle$ Referencia del artículo}

Díaz-Vásquez, A. R., Romero-de De la Vega, R. R.,Rodas-Escobar, D. M., Ramírez-Cortez, A. M., Cabrera-Morales, M. de J (2001). La innovación de las bibliotecas. Revista Guatemalteca de Cultura. 1(1),10-16. DOI: 10.46954/revistaguatecultura.v1i1.6

Recibido 15/09/ 2020

Aceptado 25/01/2021

\section{Resumen}

El presente artículo resalta la importancia que tiene las innovaciones tecnológicas en el que hacer de las bibliotecas, mismas que fueron acondicionadasenelúltimo año. Esimportantereconocer, quelasbibliotecas antes de la pandemia siempre fueron consideradas como lugares que guardaban y conservaban el acervo bibliográfico de la humanidad y que en algunas ocasiones, hacían uso de las nuevas tecnologías para prestar 
algún servicio al usuario; actualmente este concepto ha evolucionado, pues las bibliotecas se han convertido en lugares que ofrecen formación e información de manera gratuita, convirtiéndose en lugares dedicados a cubrir las necesidades curriculares y de información del usuario, pero también ofrecen formación técnico-científica, con el aprendizaje de la alfabetización mediática informacional, pues se capacita al usuario para que acceda a la información, pero además que tenga conciencia de que todo cuanto hace y comparte en las redes sociales, es susceptible a ser visto por muchas personas, por lo que debe ser responsable de lo que lee, comparte y a lo que le da me gusta.

$\triangle$ Palabras clave: biblioteca digital, innovación en biblioteca, recursos digitales

\section{Abstract}

This article highlights the importance of technological innovations, in the activities of the libraries, innovations that were refurbished in the last year. It is important to recognize that libraries, before the pandemic, were always considered as places that store and conserve the bibliographic heritage of humanity and that in some occasions, they used the new technologies to provide some services to the user; currently this concept has evolved, as libraries have become places that offer training and information for free, becoming places dedicated to fulfill the curricular and informational needs of the user, but also they offer technical-scientific training, with the learning of informational media literacy, since the user is trained to access the information, but also to be aware that everything he does and shares in social networks is susceptible of being seen by many people, so he must be responsible for what he reads, shares and likes.

Keywords: digital library, library innovation, digital resources 


\section{Introducción}

El presente artículo se elabora con el fin de abordar el impacto que han tenido las innovaciones tecnológicas en el último año, en los servicios de difusión del conocimiento entre los usuarios de las bibliotecas. Actualmente las bibliotecas son lugares que ofrecen formación e información de manera gratuita y que atienden a los usuarios al proveerles de actividades de entretención y esparcimiento.

Debido a los cambios de conducta, a las exigencias y necesidades de los usuarios las bibliotecas están empezando a migrar de bibliotecas tradicionales a bibliotecas digitalizadas, la era digital está obligando a los bibliotecarios a adaptarse a estos cambios tecnológicos. Con estas innovaciones tecnológicas, los recursos que originalmente eran en forma impresa, en la actualidad se ofrecen en formato digital, haciendo que la biblioteca deje de ser el lugar en donde se obtiene información en forma pasiva, convirtiéndose en un lugar proactivo, capaz de integrar a los miembros de una comunidad en actividades dinámicas, para que los usuarios se conviertan en socios activos, que impulsen el desarrollo y el cambio en su comunidad (Bargiela, et al., 2007).

Con las innovaciones tecnológicas las bibliotecas se han tenido que reinventar, dando un paso hacia adelante y usando las nuevas tecnologías como herramientas para satisfacer las necesidades de los usuarios. Las bibliotecas han dejado de ser centros de resguardo de la información para convertirse en centros modernos que prestan servicios a través de la tecnología, brindando información que se obtiene de internet en las redes sociales, en libros digitales y en repositorios institucionales (Bogado y Dapozo, s.f.).

Los bibliotecólogos han adquirido nuevos roles y están comprometidos con nuevos campos de acción, pues ahora su rol principal es el de facilitador de la información es decir que para implementar bibliotecas virtuales se aplican innovaciones tecnológicas para convertir la biblioteca tradicional a virtual, digital o electrónica, donde la colección que contiene documentos digitales se organiza según las necesidades del usuario, cumpliendo con los procesos técnicos básicos como: selección, clasificación, catalogación, indización y digitalización.

Para las bibliotecas que se están actualizando y transformando ofrecen acceso a la información, atendiendo necesidades que satisfacen los intereses de los usuarios, implementando servicios especiales, como lo recomienda Voutssas (2006) con espacios "cómodos, cubículos placenteros, colaborar con las empresas que soliciten información y ofrecer talleresdecapacitación parael públicoengeneral que sean proporcionados por instructores profesionales y voluntarios de la comunidad", además Alonso-Arévalo y Cordón-García (2015) consideran que "las bibliotecas están sirviendo de catalizador para un cambio en la misión, visión, objetivos y servicios que desde hace tiempo ya venían desarrollando propuestas acordes al interés de sus comunidades" ya que "países desarrollados como Estados Unidos, Reino Unido, Francia y Suecia, forman parte de la generación \#hashtag" (parr. 1-4), por tanto para ellos es muy fácil acceder a todo lo digital. 
Las bibliotecas utilizan como herramientas las nuevas tecnologías para ofrecer servicios innovadores para toda la gama de usuarios que atienden, permitiendo así se acorte la brecha digital pues resuelven consultas desde cualquier lugar, valiéndose de otras disciplinas científicas como la pedagogía y la informática para formarlos y así facilitar la comunicación y el aprendizaje, generando nuevas competencias.

Actualmente los proveedores de publicaciones periódicas distribuyen su material por medio de bases de datos, para ser usadas en las bibliotecas por medio de Internet y ofreciendo el uso de la información a sus usuarios por medio de FTP- File Transfer Protocol, permitiendo el intercambio de archivos entre computadoras, y facilitando que los científicos intercambien datos entre colegas.

\section{Materiales y métodos}

La metodología que se utilizó es documental. Se dio lectura como primera fuente de consulta a artículos científicos de acceso libre publicados en revistas científicas, escritos por autores del área de bibliotecología como por ejemplo Alonso y Cordón; Bargiela y colaboradores; Bogado y Dapozo y Voutssas.

\section{Resultados}

En el presente artículo se describe cómo fue el auge de las nuevas tecnologías de información y comunicación y cómo las bibliotecas tuvieron que innovar sus procesos, como por ejemplo los catálogos, los índices, los repositorios,

las revistas científicas; haciendo uso de herramientas tecnológicas para ofrecer recursos digitales a sus usuarios. Un cambio que han tenido las bibliotecas, es que los presupuestos se han orientado más a la adquisición de documentos digitales, teniendo que prescindir o disminuir en cantidad las comprar impresas.

Sin embargo ha sido difícil también para las bibliotecas mantenerse a la vanguardia para dar servicios gratuitos a los usuarios como es el caso de la Biblioteca Central de la Universidad de San Carlos de Guatemala, que invierte recursos económicos para ofrecer en su página de Internet acceso libre a prestigiosos catálogos, como la plataforma EBSCO, E-libro, AGORA, OARE, LATINDEX, Biblioteca Digital Hispánica, Biblioteca del Banco Mundial, Biblioteca de la Organización Panamericana de la Salud y la Biblioteca del Fondo Monetario International.

Debido al crecimiento de la producción científica y literaria, en Estados Unidos como en el Reino Unido las ventas de libros electrónicos superan a las de los libros impresos por lo que las colecciones digitales han aumentado considerablemente, y esto ha contribuido a que la circulación, o sea los préstamos o consultas digitales también hayan aumentado, según se lee a continuación.

Alonso y Cordón, (2015) señalan que: . . 
el año 2013. Los datos para sistemas de bibliotecas ubicadas en poblaciones de 500,000 o más habitantes son aún mayores con un promedio que supera los 30,000 títulos prestados. Seis bibliotecas estadounidenses han superado el millón de préstamos, llegando a los 102 millones el año pasado, según datos de Overdrive, la mayor plataforma de préstamo digital del mundo. (p. 4)

\section{Discusión}

Para dar respuesta a la pregunta ¿para qué servirá la biblioteca digital a los diferentes actores de las comunidades en el futuro? Principalmente para cerrar la brecha digital, ofreciendo servicios digitales eficientes a los usuarios que consultan la biblioteca, por medio de la integración de los siguientes elementos: la comunicación entre las personas fomentando las relaciones interpersonales que permitan fortalecer el capital humano; el uso de espacios virtuales y plataformas tecnológicas que potencien e innoven su aprendizaje y educación, y que a la vez fomenten la socialización de las ideas y los conocimientos de la comunidad.

Por todo esto es que las bibliotecas deben de mantenerse actualizadas y así cumplir las necesidades digitales de información de los usuarios, para poder cumplir con la función de ser facilitadoras de información y creadoras de conocimiento por medio de los recursos digitales que actualmente ofrecen.
Para utilizar las innovaciones tecnológicas que les permitan a las bibliotecas ofrecer recursos y servicios digitales a los usuarios, sin llegar a las bibliotecas, a través de los diferentes medios de comunicación, no es necesario contar con un gran presupuesto, plataformas caras, máquinas muy completas o el mejor edificio; para que una biblioteca sea digital e innovadora y brinde este tipo de servicios a sus usuarios, se requiere únicamente de la visión futurista de los bibliotecarios a cargo de las Unidades de Información; que estén dispuestos a ofrecer sus servicios a través de las distintas plataformas gratuitas como lo son Facebook, Google drive, Instagram, Telegram y otras, y que pueden albergar de manera gratuita libros y artículos de revistas de acceso libre, facilitando la recuperación de la información a los usuarios.

Entonces los desafíos que enfrentan las bibliotecas para los próximos años se centran en tres puntos esenciales: a) invertir en formatos digitales; b) repensar los modelos de servicios; y c) el fortalecimiento de la alianza entre los editores y productores de contenido y las redes de comunicación para facilitar el acceso a la información digital a los usuarios. 


\section{Referencias}

Alonso-Arevalo, J. y Cordón-García, A. (2015). ¿Para qué servirá la Biblioteca Pública en el futuro? Depende de su capacidad de adaptación a los imparables cambios sociales, económicos y tecnológicos.? DBD. Artículos del Departamento de Biblioteconomía y Documentación. https://gredos.usal.es/bitstream/handle/10366/125206/ MiBibliotecaNNTT-MB401.pdf?sequence $=1$

Bargiela, R., Dapozo, G. N., y Bogado, V. S. (2007). Digitalización de documentos para su utilización en una biblioteca virtual. In II Congreso de Tecnología en Educación y Educación en Tecnología. http://sedici.unlp.edu.ar/bitstream/handle/10915/19150/31. pdf?sequence $=1$

Bogado, G., y Dapozo, B. R. (s.f.) Digitalización de documentos para su utilización en una biblioteca virtual. Departamento de Informática. Facultad de Ciencias Exactas y Naturales y Agrimensura. Universidad Nacional del Nordeste.

http://sedici.unlp.edu.ar/bitstream/handle/10915/19150/Documento_ completo.pdf?sequence $=1 \&$ isAllowed $=y$

Voutssás, J. (2006). Bibliotecas y publicaciones digitales. Universidad

Nacional Autónoma de México. https://libros.metabiblioteca.org/jspui/ bitstream/001/345/4/970-32-3962-5.pdf 


\section{Sobre las autoras}

Equipo de investigadoras de la Maestría en Bibliotecología y Ciencia de la Información de la Universidad de San Carlos de Guatemala.

Copyright (c) Ana Ruth Díaz y colaboradoras

\section{Este texto está protegido por una licencia Creative Commons 4.0.}

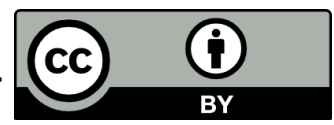

Usted es libre para compartir, copiar y redistribuir el material en cualquier medio o formato y adaptar el documento, remezclar, transformar y crear a partir del material para cualquier propósito, incluso comercialmente, siempre que cumpla la condición de atribución: usted debe reconocer el crédito de una obra de manera adecuada, proporcionar un enlace a la licencia, e indicar si se han realizado cambios. Puede hacerlo en cualquier forma razonable, pero no de forma tal que sugiera que tiene el apoyo del licenciante o lo recibe por el uso que hace. 